\title{
Minimizing leukemia escape: implementing a dual anti-CD20- and CD19-scFv-based chimeric antigen receptor (CAR)
}

\author{
Dina Schneider ${ }^{1 *}$, Ying Xiong ${ }^{1}$, Andre Roy ${ }^{1}$, Andrew Kaiser ${ }^{2}$, Boro Dropulic ${ }^{1}$, Rimas Orentas ${ }^{1}$ \\ From 30th Annual Meeting and Associated Programs of the Society for Immunotherapy of Cancer (SITC 2015) \\ National Harbor, MD, USA. 4-8 November 2015
}

Adoptive immunotherapy with chimeric antigen receptor (CAR) transduced $\mathrm{T}$ lymphocytes has shown promising results in both pediatric and adult $B$ cell malignancies. Nevertheless, both CAR-based and antibody-based antiCD19 therapies, e.g. blinatumomab, have seen treatment failures attributed to the loss of CD19 or an epitope of CD19 on the surface of the malignant B cell. It may be possible to overcome antigen escape by targeting two tumor antigens simultaneously, i.e. CD19 and CD20 using a tandem construct with two scFv-based CAR binding domains. Lentiviral vectors encoding chimeric antigen receptors comprised of anti-CD19 and antiCD20 targeting domains expressed alone or in tandem were transduced into $\mathrm{T}$ cells from healthy donors to generate the corresponding CAR19, CAR20, CAR19_20 (CD19 scFv more distal to the $\mathrm{T}$ cell plasma membrane) and CAR20_19 T cells (CD20 scFv distal). The transduced $\mathrm{T}$ cells were $50-70 \%$ CAR positive as determined by protein L flow cytometric analysis. Expression of CAR proteins of the expected molecular weight was confirmed by Western blot analysis of transduced T cells. When CAR-transduced T cells were combined with CD19 ${ }^{+} \mathrm{CD} 20^{+}$Raji target cells, but not $\mathrm{CD} 19^{-} \mathrm{CD} 20^{-} \mathrm{K} 562$ cells, all four CAR $\mathrm{T}$ cell types demonstrated comparable efficient killing of leukemia targets (E:T ratio $>2$ ), and target-dependent induction of IFN- $\gamma$, as measured in coculture supernatants by ELISA.

We then began a series of in vitro co-culture experiments where we used very low E:T ratios to examine the potential for CAR-induced antigen loss on surviving leukemia cells. All CAR-T cells expressing an anti-CD19 $\mathrm{scFv}$ induced rapid loss of CD19 surface expression.
In contrast, the CD20 surface marker was less prone to down-regulation by CAR-T cells expressing anti-CD20 $\mathrm{scFv}$. Upon flow cytometric analysis of surviving leukemia cells on day 5, CD19 expression was reduced to $3 \%$, $48 \%, 73 \%, 90 \%$ and $93 \%$ of control when co-cultured with CAR T cells expressing CAR19_20, CAR20_19, CAR19, CAR20, and control T cells, respectively. Similar results were seen when experiments were of longer, 7 days, or shorter, 1 day, duration. In conclusion, tandem CAR T cells are as effective as single CAR19 or CAR20 $\mathrm{T}$ cells in leukemia cell killing. Importantly, dual scFvexpressing CARs are more potent in preventing tumor antigen escape via target antigen down-regulation.

\section{Authors' details}

'Lentigen Technology Inc., Gaithersburg, MD, USA. ${ }^{2}$ Miltenyi Biotec GmbH, Bergisch Gladbach, Germany.

Published: 4 November 2015

doi:10.1186/2051-1426-3-S2-P122

Cite this article as: Schneider et al:: Minimizing leukemia escape:

implementing a dual anti-CD20- and CD19-scFv-based chimeric antigen receptor (CAR). Journal for ImmunoTherapy of Cancer 2015 3(Suppl 2):P122.

'Lentigen Technology Inc., Gaithersburg, MD, USA

Full list of author information is available at the end of the article 